\title{
Reevaluation of Neutron Electric Dipole Moment with QCD Sum Rules
}

\author{
hisano \\ Department of Physics, \\ Nagoya University \\ Nagoya 464-8602, Japan \\ E-mail: hisano@eken.phys.nagoya-u.ac.jp
}

\begin{abstract}
We study the neutron electric dipole moment in the presence of the CP-violating operators up to the dimension five in terms of the QCD sum rules. By using input parameters obtained from the lattice calculation, we derive a conservative limit for the contributions of the $\mathrm{CP}$ violating operators.
\end{abstract}

Xth Quark Confinement and the Hadron Spectrum,

October 8-12, 2012

TUM Campus Garching, Munich, Germany 


\section{Introduction}

The electric dipole moment (EDM) of neutron is one of the physical quantities that are quite sensitive to the CP violation in the flavor-conserving interaction. Since there has been no experimental evidence for its existence so far, a severe constraint is imposed on the CP-violating interactions. The currently most stringent limit for the neutron EDM is given by the Institut LaueLangevin (ILL) experiment [1]: $\left|d_{n}\right|<2.9 \times 10^{-26} e \mathrm{~cm}$ (90\% C.L.). Moreover, several experimental projects which use ultra cold neutrons are now under development and expected to have much improved sensitivities. Such high sensitivities provide an opportunity to probe the flavorconserving CP-violating interactions in the TeV-scale physics beyond the SM. Furthermore, we may probe the flavor violation in the new physic indirectly. Even if the new flavor-violating interactions are introduced in the new physics, the relative $\mathrm{CP}$ phase between them and the CKM matrix may contribute to the EDM [2].

In order to translate the experimental limits for the neutron EDM into constraints on the CP violation on the Lagrangian at parton level, one needs to obtain a relation between these two quantities. There are some attempts to derive the relation based on the naive dimensional analysis, the chiral perturbation theory, and the QCD sum rules, though they are considered to have large uncertainties. It is ultimately desired that the lattice QCD simulation would evaluate it in future.

In this contribution, we show the evaluation of the neutron EDM with the QCD sum rules, including the CP-violating operators up to the dimension five, which is derived in Ref. [3]. Similar attempts have been already made in the previous works, e.g., in a series of papers by M. Pospelov and A. Ritz [4, 5] and references therein. We also derive the sum rules for the neutron EDM, while we use the lattice QCD simulation result for the low-energy constant in the numerical evaluation of the neutron EDM. It is found that this gives more conservative estimate than carrying out all of the evaluation within the framework of the QCD sum rules. This approach provides a way of eliminating theoretical errors from the calculation, while there still remains uncertainty resulting from the QCD sum rule technique itself.

This article is organized as follows. In Sec. 2, we review the CP-violating interactions at parton level up to the dimension five. From Sec. 3, the analysis of the neutron EDM with the QCD sum rules starts. In Sec. 3 we discuss phenomenological aspects of the correlator of the interpolating field to neutron, and, in Sec. 4, show the properties of the neutron-interpolating field. In Sec. 5 we extract the low-energy constant from the lattice QCD simulation result. In Sec. 6 our numerical results for the neutron EDM are derived. Section 7 is devoted to conclusion. Since the pages for this contribution are limited, we do not write evaluation of the operator product expansion (OPE) for the correlator on the CP-violating and electromagnetic background. See Ref. [3] if you would like to know it.

\section{Effective Lagrangian}

Let us first express the flavor-conserving CP-violating terms in the low-energy effective Lagrangian for the system consisting of light quarks and gluon. We include all of the CP-violating 
operators up to the dimension five:

$$
\begin{aligned}
\mathscr{L}_{d P}= & -\sum_{q=u, d, s} m_{q} \bar{q} i \theta_{q} \gamma_{5} q+\theta_{G} \frac{\alpha_{s}}{8 \pi} G_{\mu \nu}^{A} \tilde{G}^{A \mu \nu} \\
& -\frac{i}{2} \sum_{q=u, d, s} d_{q} \bar{q}(F \cdot \sigma) \gamma_{5} q-\frac{i}{2} \sum_{q=u, d, s} \tilde{d}_{q} \bar{q} g_{s}(G \cdot \sigma) \gamma_{5} q .
\end{aligned}
$$

Here, $m_{q}$ represents the quark masses, $F_{\mu \nu}$ and $G_{\mu \nu}^{A}$ are the electromagnetic and gluon field strength tensors, $g_{s}$ is the strong coupling constant. The second, third and forth terms in Eq. (2.1) are called the effective QCD $\theta$ term, the electric and chromoelectric dipole moments (CEDMs) for quarks, respectively. The EDMs and CEDMs for quarks are dimension-five operators, and they are sensitive to the $\mathrm{TeV}$-scale physics beyond the SM. They are evaluated by integrating out the heavy particles and evaluating the renormalization-group equation effects to the coefficients ${ }^{1}$. The coefficients of the CP-violating operators, $\theta_{q}, \theta_{G}, d_{q}$, and $\tilde{d}_{q}$, are all assumed to be quite small, and we keep only the terms up to the first order of these parameters. The first two terms in Eq. (2.1) are mutually related by the chiral rotation. Under the following infinitesimal chiral rotation,

$$
q \rightarrow q^{\prime}=\left(1-i \varepsilon \rho_{q} \gamma_{5}\right) q
$$

where $\rho_{q}=\theta_{q} / \theta_{Q}\left(\theta_{Q} \equiv \sum_{q=u, d, s} \theta_{q}\right), \bar{\theta}=\theta_{G}+\theta_{Q}$ is invariant so that it is a physical parameter.

There remains still some arbitrariness in the quark mass phases $\theta_{q}$, since they are redefined into another through an $S U(3)$ chiral rotation. In this article, we choose an appropriate set of $\theta_{q}$ so that the choice significantly reduces the CP-violating contribution to the vacuum expectation values (VEVs) of quark bi-linear. We take the condition in Ref. [7] to determine $\theta_{q}$, that is, after the $\theta$ term rotated into the $\gamma_{5}$-mass term, $\left\langle\Omega_{\mathrm{CP}}\left|\mathscr{L}_{\mathrm{CP}}\right| M^{A}\right\rangle=0\left(M^{A}=\pi, K, \eta\right)$ should be satisfied. This condition is evaluated by using the partially conserved axial-vector current (PCAC) relations. With the relation $\sum_{q} \rho_{q}=1$, we then determine the quark mass phases as follows:

$$
\rho_{q}=\frac{m_{*}}{m_{q}}\left[1+\frac{m_{0}^{2}}{2 \bar{\theta}}\left\{\frac{\tilde{d}_{q}-\tilde{d}_{q^{\prime}}}{m_{q^{\prime}}}+\frac{\tilde{d}_{q}-\tilde{d}_{q^{\prime \prime}}}{m_{q^{\prime \prime}}}\right\}\right]
$$

where $1 / m_{*} \equiv 1 / m_{u}+1 / m_{d}+1 / m_{s}$ and $\left\langle\bar{q} g_{s}(G \cdot \sigma) q\right\rangle=-m_{0}^{2}\langle\bar{q} q\rangle$.

\section{Phenomenological behavior of correlator}

The QCD sum rules are based on an analysis of the correlator of interpolating fields. In the method, OPE allows one to consistently separate the long- and short-distance contributions to the correlator, and the long-distance contributions are evaluated by condensations of quarks and gluon. By comparing the evaluated correlator with the phenomenological model, the properties for the low-lying parts of the hadronic spectrum are derived. The Borel transformation is applied to the correlator there. In this section, we first discuss the phenomenological model for the correlator.

In the present case, the interpolating field must have the same quantum numbers as those of neutron, and it is denoted by $\eta_{n}(x)$ hereafter. On a background with CP-violating sources, the

\footnotetext{
${ }^{1}$ The renormalization-group equations of the Wilson coefficients for the CP-violating interaction up to dimension six at one-loop level is completed recently [6].
} 
matrix element of the interpolating field between the vacuum $\left|\Omega_{d P}\right\rangle$ and the one-particle neutron state on the CP-violating background $\left|N_{C P}(\mathbf{p}, \mathbf{s})\right\rangle$ is given as

$$
\left\langle\Omega_{C P}\left|\eta_{n}(x)\right| N_{d P}(\mathbf{p}, s)\right\rangle=\lambda_{n} e^{\frac{i}{2} \alpha_{n} \gamma_{5}} u_{n}(\mathbf{p}, s) e^{-i p \cdot x} .
$$

with $u_{n}(\mathbf{p}, s)$ an ordinary spinor wave function which satisfies $\left(\not p-m_{n}\right) u_{n}(\mathbf{p}, s)=0$. The phase factor $e^{-i \alpha_{n} \gamma_{5}}$ appears due to the $\gamma_{5}$ mass term for neutron. The low-energy constant $\lambda_{n}$ is to be determined later.

Now we analyze the correlator of the interpolating fields from the phenomenological viewpoint. It is defined as

$$
\Pi(q) \equiv i \int d^{4} x e^{i q \cdot x}\left\langle\Omega_{d P}\left|T\left\{\eta_{n}(x) \bar{\eta}_{n}(0)\right\}\right| \Omega_{d P}\right\rangle_{F},
$$

where the subscript $F$ implies that the correlator is evaluated on an electromagnetic field background. Our goal is to extract the EDM of neutron from the correlator. The phase factor in Eq. (3.1), however, causes mixture between electric and magnetic dipole moment structures and makes it difficult to pick out only the EDM from the QCD sum rules. So we first examine the Lorentz structures of the correlator and select a term independent of the phase $\alpha_{n}$, i.e., chiral invariant. As discussed in Ref. [4], up to the leading order on the background electromagnetic field, the correlator $\Pi(q)$ is estimated by inserting an effective vertex such as $\mathscr{L}_{n}=-(i / 2) d_{n} \bar{N}(F \cdot \sigma) \gamma_{5} N$. Here, $N(\equiv N(x))$ denotes the renormalized neutron field which is approximately equivalent to $\lambda_{n}^{-1} e^{-i \alpha_{n} \gamma_{5} / 2} \eta_{n}(x)$, and $d_{n}$ is the EDM of neutron. A similar procedure to those in Ref. [4] shows that terms with an odd number of Dirac matrices are independent of the phase factor $\alpha_{n}$, and furthermore, those proportional to $\{\tilde{F} \cdot \sigma, \not\}$ are the unique choice in this case. Therefore we only focus on such terms in the following calculation. Then, the phenomenological expression of the correlator is found to be.

$$
\Pi^{\text {(phen })}(q)=\frac{1}{2} f\left(q^{2}\right)\{\tilde{F} \cdot \sigma, q\}+\ldots,
$$

where dots indicate terms with other Lorentz structures and

$$
f\left(q^{2}\right)=\left(\frac{\lambda_{n}^{2} d_{n} m_{n}}{\left(q^{2}-m_{n}^{2}\right)^{2}}+\frac{A\left(q^{2}\right)}{q^{2}-m_{n}^{2}}+B\left(q^{2}\right)\right)
$$

with $A\left(q^{2}\right)$ and $B\left(q^{2}\right)$ functions which have no pole at $q^{2}=m_{n}^{2}$. As noted in Ref. [4], since we are effectively dealing with a three-point function, it might be inconsistent to parametrize the continuum contribution in terms of a usual ansatz for the spectral function with a certain threshold in the QCD sum rules. We just neglect the contribution with expecting its significance to be small enough. Furthermore, we assume that the function $A\left(q^{2}\right)$ has little dependence on $q^{2}$, and regard it as a constant when we conduct the Borel transformation.

\section{Neutron-interpolating field}

In this section we give discussion on choice of the neutron-interpolating field which we use for the QCD sum rule calculation. The field must have the same quantum numbers as neutron. The most general interpolator for neutron on the ordinary $\mathrm{CP}$-even background is parametrized as

$$
\eta_{n}(x)=j_{1}(x)+\beta j_{2}(x),
$$


where $j_{1}(x)=2 \varepsilon_{a b c}\left(d_{a}^{T}(x) C \gamma_{5} u_{b}(x)\right) d_{c}(x)$ and $j_{2}(x)=2 \varepsilon_{a b c}\left(d_{a}^{T}(x) C u_{b}(x)\right) \gamma_{5} d_{c}(x)$. Here the subscripts, $a, b, c$, denote the color indices and $C$ is the charge conjugation matrix. The interpolator $j_{1}(x)$ is often used in lattice simulations.

When the calculation is carried out on the CP-violating background, however, the interpolating fields include additional components. This point is easily understood when one considers the chiral rotation discussed in Sec. 2. As we have seen in Sec. 2, the chiral rotation (2.2) transforms the Lagrangian $\mathscr{L}$ into another. The same transformation, in turn, changes the interpolators $j_{1}(x)$ and $j_{2}(x)$ as $j_{1}(x) \rightarrow j_{1}(x)-i \varepsilon\left[\left(\rho_{u}+\rho_{d}\right) i_{1}(x)+\rho_{d} i_{2}(x)\right]$ and $j_{2}(x) \rightarrow j_{2}(x)-i \varepsilon\left[\left(\rho_{u}+\right.\right.$ $\left.\left.\rho_{d}\right) i_{2}(x)+\rho_{d} i_{1}(x)\right]$, respectively, where $i_{1}(x)=\gamma_{5} j_{2}(x)$ and $i_{2}(x)=\gamma_{5} j_{1}(x)$. After all, the neutroninterpolating field which we deal with has a following structure:

$$
\eta_{n}(x)=j_{1}(x)+\beta j_{2}(x)+i \varepsilon\left[i_{1}(x)+\beta i_{2}(x)\right] .
$$

The small constant $\varepsilon$ is determined by the condition that the interpolating field $\eta_{n}(x)$ has a vanishing correlator with the current $\xi_{n}(x)\left(\equiv i_{1}(x)+\beta i_{2}(x)+i \varepsilon\left[j_{1}(x)+\beta j_{2}(x)\right]\right)$. In what follows, however, we sweep away the contribution of the mixture terms in the interpolating field by choosing an appropriate value for the parameter $\beta$. As discussed in Sec. 3, we focus on parts of the correlators which have the Lorentz structures with an odd number of gamma matrices. Such terms anti-commute with $\gamma_{5}$. Thus, in this case, the above expression leads to

$$
\begin{aligned}
\left.\left\langle\Omega_{\mathrm{dP}}\left|T\left\{\eta_{n}(x) \bar{\eta}_{n}(0)\right\}\right| \Omega_{\mathrm{dP}}\right\rangle_{F}\right|_{\gamma_{\text {odd }}}= & \left\langle j_{1}, \bar{j}_{1}\right\rangle+\beta\left[\left\langle j_{1}, \bar{j}_{2}\right\rangle+\left\langle j_{2}, \bar{j}_{1}\right\rangle\right]+\beta^{2}\left\langle j_{2}, \bar{j}_{2}\right\rangle \\
& +i \varepsilon\left(1-\beta^{2}\right)\left[\left\langle j_{1}, \bar{j}_{2}\right\rangle-\left\langle j_{2}, \bar{j}_{1}\right\rangle\right] \gamma_{5}
\end{aligned}
$$

where

$$
\langle a, \bar{b}\rangle \equiv\left\langle\Omega_{\mathrm{CP}}|T\{a(x) \bar{b}(0)\}| \Omega_{\mathrm{CP}}\right\rangle_{F} .
$$

This equation shows that the mixing terms in the interpolating field do not affect the correlator if one sets $\beta$ to be \pm 1 . $\beta=+1$ is an appropriate choice since this choice eliminates the sub-leading terms with infrared logarithm in OPEs, which yield ambiguity due to the infrared cutoff. With this choice one may simultaneously exclude the contribution of the mixing terms. Thus, we will not calculate such mixing contributions with keeping in mind that we will finally take $\beta=+1$ when we derive the QCD sum rules.

\section{Determination of $\lambda_{n}$ from lattice}

The low-energy constant $\lambda_{n}$ determines the normalization of the QCD sum rules so that the uncertainties are directly linked to the final result. We extract its numerical value from the lattice QCD calculation presented in Ref. [8], in which the QCD matrix elements for the proton decay rate are evaluated. In fact, they evaluate a similar quantity for proton, though the isospin symmetry allows us to interpret it for the present purpose. After translating the above value of $\lambda_{n}$ in Ref. [8] into that of at the renormalization scale $\mu=1 \mathrm{GeV}$, we obtain

$$
\lambda_{n}=-0.0436 \pm 0.0047_{\text {(stat) }} \pm 0.0084_{(\text {syst })} \mathrm{GeV}^{3}
$$

for $\beta=+1$. 
Let us compare the value of $\lambda_{n}$ obtained here with those used in the previous works. In Ref. [4], for example, they exploit the values for $\lambda_{n}$ evaluated in Ref. [9] by using the QCD sum rules. Two Dirac- $\gamma$ structures, $\mathbb{1}$ and $\not p$, provide different sum rules. As evaluated in Ref. [9], these two sum rules yield relatively small values for $\lambda_{n}$; the lattice QCD value is several times larger than the values evaluated by using the QCD sum rules. The author in Ref. [9] also estimates the error for these values. It is about $30 \%$ for the sum rules result, while $20 \%$ for the lattice QCD result. The lattice QCD result might have a uncertainty in the chiral extrapolation. Since there is no more guiding principle for judging which estimation is valid, we exploit the lattice QCD result in Eq. (5.1) because this choice leads to rather conservative constraint for CP-violating sources.

\section{Results}

In order to derive the QCD sum rules for the present case, we first extract the coefficient functions of $\{\tilde{F} \cdot \sigma, q\}$ from both the phenomenological and the OPE correlators, $\Pi^{(\text {phen })}$ and $\Pi^{(\mathrm{OPE})}$. The phenomenological side is derived from Eq. (3.4) as

$$
C^{(\text {phen })}\left(Q^{2}\right) \equiv \frac{1}{2}\left[\frac{\lambda_{n}^{2} d_{n} m_{n}}{\left(Q^{2}+m_{n}^{2}\right)^{2}}-\frac{A}{Q^{2}+m_{n}^{2}}\right]
$$

while the OPE side at the next-to-leading order is given in Ref. [3] as

$$
C^{(\mathrm{OPE})}\left(Q^{2}\right) \equiv \frac{1}{16 \pi^{2}}\langle\bar{q} q\rangle \Theta \log \left(\frac{Q^{2}}{\Lambda^{2}}\right)
$$

with $Q^{2} \equiv-q^{2}$ and

$$
\Theta \equiv\left(4 e_{d} m_{d} \rho_{d}-e_{u} m_{u} \rho_{u}\right) \chi \bar{\theta}+\left(4 d_{d}-d_{u}\right)+\left(\kappa-\frac{1}{2} \xi\right)\left(4 e_{d} \tilde{d}_{d}-e_{u} \tilde{d}_{u}\right)
$$

In Eq. (6.1), we neglect the continuum contribution and think of $A$ as a constant, as discussed above. By equating the coefficient functions after the Borel transformation, we finally derive the sum rules as follows:

$$
\lambda_{n}^{2} d_{n} m_{n}-A M^{2}=-\Theta\langle\bar{q} q\rangle \frac{M^{4}}{8 \pi^{2}} e^{\frac{m_{n}^{2}}{M^{2}}},
$$

with $M$ so-called the Borel mass. All we have to do is now reduced to determining the Borel mass $M$ and the coupling $\lambda_{n}$, as well as estimating the parameter $A$.

Now we estimate the neutron EDM by using the results obtained above. First of all, we rewrite the sum rules in Eq. (6.4) in a simple form:

$$
c_{0}+c_{1} x=f(x)
$$

where $x=M^{2}$ and

$$
f(x) \equiv \frac{x^{2}}{8 \pi^{2}} \exp \left(\frac{m_{n}^{2}}{x}\right), \quad c_{0} \equiv \frac{d_{n} \lambda_{n}^{2} m_{n}}{-\Theta\langle\bar{q} q\rangle}, \quad c_{1} \equiv \frac{-A}{-\Theta\langle\bar{q} q\rangle} .
$$

The right-hand side of Eq. (6.5) describes the behavior of the coefficient function obtained from the OPE calculation, while the left-hand side represents the phenomenological one. The first and 
second terms in the left-hand side correspond to the double and single pole contributions, i.e., the first and second terms in Eq. (3.4), respectively. Once given a Borel mass point $x=M^{2}$, one may readily pick out $c_{0}$ and $c_{1}$ from the tangent line to the function $f(x)$ at the point. We here assume our sum rules to be valid within the region of the Borel mass in which the single pole contribution is less than $30 \%$ of the double pole contribution. This assumption leads to $0.36 \mathrm{GeV}^{2}<M^{2}<0.50 \mathrm{GeV}^{2}$, and in this region, $d_{n}$ takes $d_{n}=2.4_{-0.3}^{+0.6} \times 10^{-1} \Theta$, where the lower value corresponds to the upper limit of the Borel mass, and vice versa. Here we take $\langle\bar{q} q\rangle \simeq-(0.280 \mathrm{GeV})^{3}$.

Next, we discuss the uncertainty of the OPE calculation. In this case, the truncation of the OPE leads to the uncertainty. Let us estimate it by evaluating the relative size of the higher-order contributions. Among them, the four-quark condensates such as $\langle\bar{q} q \bar{q} q\rangle$ are expected to yield sizable contributions, since they are free from loop suppression. On the assumption that these contributions vanish when one takes the quark masses to be zero, we expect that they are suppressed at least a factor of $\langle\bar{q} q\rangle^{\frac{2}{3}} / M^{2} \simeq 0.1$. Therefore, the uncertainty of the OPE calculation is estimated to be $\mathscr{O}(10) \%$.

Taking the above discussion into account, we finally evaluate the neutron EDM with theoretical uncertainty as follows:

$$
d_{n}=2.4_{-0.3}^{+0.6} \pm 0.1_{-0.4}^{+0.7} \times 10^{-1} \Theta,
$$

where the first uncertainty stems from the phenomenological calculation while the second one comes from the approximation in the OPE. We also include uncertainties originate from those in $\lambda_{n}$ (See Eq. (5.1).), which is indicated by the third error in the above equation. After all, it is found that there is almost $\mathscr{O}(1)$ factor of uncertainty in our sum rule calculation.

For one's convenience, we substitute the numerical values for the QCD parameters in Eq. (6.7). Here we take $m_{0}^{2}=0.8 \mathrm{GeV}^{2}, \chi=-5.7 \pm 0.6 \mathrm{GeV}^{-2}, \xi=-0.74 \pm 0.2$, and $\kappa=-0.34 \pm 0.1$ $[10,11]$. Then, with those parameters the center values, we find

$$
d_{n}=8.2 \times 10^{-17} \bar{\theta}[e \mathrm{~cm}]-0.23 d_{u}+0.95 d_{d}+e\left(-0.37 \tilde{d}_{u}+0.37 \tilde{d}_{d}-0.02 \tilde{d}_{s}\right) .
$$

The contributions from $\bar{\theta}$ and the quark CEDMs to $d_{n}$ may be changed furthermore by about $\pm 10 \%$, mainly due to the theoretical uncertainty of $\chi$.

It is known that $O(1) \bar{\theta}$ induces too large neutron EDM, the strong CP problem. The PecceiQuinn (PQ) symmetry is one of the solutions for the strong CP problem. If the PQ symmetry is introduced, $\bar{\theta}$ vanishes dynamically. However, if the quark CEDMs are non-vanishing, the $\theta$ term is induced as $\bar{\theta}_{\text {ind }}=\frac{m_{0}^{2}}{2} \sum_{q=u, d, s} \tilde{d}_{q} / m_{q}$, since the axion field have its tadpole term [12]. Taking the induced $\theta$ term into account, $\Theta$ is replaced with $\Theta^{\mathrm{PQ}}$ in Eq. 6.2,

$$
\Theta^{\mathrm{PQ}}=4 d_{d}-d_{u}+\left(\frac{m_{0}^{2}}{2} \chi+\kappa-\frac{1}{2} \xi\right)\left(4 e_{d} \tilde{d}_{d}-e_{u} \tilde{d}_{u}\right) .
$$

The contributions from the strange quark CEDM are cancelled in the presence of the PQ symmetry $[5]^{2}$. Again, we substitute the numerical values for the QCD parameters as presented in the previous section. The result is

$$
d_{n}^{\mathrm{PQ}}=-0.24 d_{u}+0.95 d_{d}+e\left(0.36 \tilde{d}_{u}+0.71 \tilde{d}_{d}\right) .
$$

\footnotetext{
${ }^{2}$ However, there is still possibility that the strange CEDM contribution to the neutron EDM may be sizable. See Ref. [13].
} 


\section{Conclusion}

We have studied the neutron EDM induced by the CP-violating interactions up to the dimensionfive operators. In order to derive the relation between the CP-violating interactions and the neutron EDM, we have used the QCD sum rule technique. There are several phenomenological parameters to estimate the relation numerically. Pospelov and Ritz also analysed the neutron EDM using the QCD sum rules $[4,5]$ and they determined the low-energy constant $\lambda_{n}$ within the framework in the QCD sum rules. On the other hand, we have extracted the $\lambda_{n}$ parameter from lattice calculations. This approach allows us to reduce a theoretical uncertainty and leads to a conservative constraint on the CP violations. Our result is about $70 \%$ smaller compared with the one obtained by Pospelov and Ritz. There still remains a sizable uncertainty resulting from the QCD sum rules itself due to a choice of the Borel mass scale. We have estimated the uncertainty from the Borel mass scale assuming that the single pole contribution is less than $30 \%$ of the double pole contributions. This assumption leads to the theoretical error of about $\mathscr{O}(1)$.

\section{References}

[1] C. A. Baker, D. D. Doyle, P. Geltenbort, K. Green, M. G. D. van der Grinten, P. G. Harris, P. Iaydjiev and S. N. Ivanov et al., Phys. Rev. Lett. 97, 131801 (2006).

[2] J. Hisano, M. Nagai and P. Paradisi, Phys. Lett. B 642, 510 (2006) ; Phys. Rev. D 78, 075019 (2008); Phys. Rev. D 80, 095014 (2009).

[3] J. Hisano, J. Y. Lee, N. Nagata and Y. Shimizu, Phys. Rev. D 85, 114044 (2012) [arXiv:1204.2653 [hep-ph]].

[4] M. Pospelov and A. Ritz, Phys. Rev. Lett. 83, 2526 (1999); Nucl. Phys. B 573, 177 (2000).

[5] M. Pospelov and A. Ritz, Phys. Rev. D 63, 073015 (2001).

[6] J. Hisano, K. Tsumura and M. J. S. Yang, arXiv:1205.2212 [hep-ph].

[7] R. J. Crewther, P. Di Vecchia, G. Veneziano and E. Witten, Phys. Lett. B 88, 123 (1979) [Erratum-ibid. B 91, 487 (1980)].

[8] Y. Aoki et al. [RBC-UKQCD Collaboration], Phys. Rev. D 78, 054505 (2008).

[9] D. B. Leinweber, Annals Phys. 254, 328 (1997).

[10] V. M. Belyaev and B. L. Ioffe, Sov. Phys. JETP 56, 493 (1982) [Zh. Eksp. Teor. Fiz. 83, 876 (1982)].

[11] I. I. Kogan and D. Wyler, Phys. Lett. B 274, 100 (1992).

[12] I. I. Y. Bigi and N. G. Uraltsev, Sov. Phys. JETP 73, 198 (1991).

[13] K. Fuyuto, J. Hisano and N. Nagata, arXiv:1211.5228 [hep-ph]. 